\title{
CARACTERIZAÇÃO DO USO DO CATETER CENTRAL DE INSERÇÃO PERIFÉRICA EM UMA UTI NEONATAL NO ESTADO DO PARANÁ
}

\author{
Ivone Costa ROSA ${ }^{1}$ \\ Gleidson Brandão OSELAME ${ }^{2}$ \\ Elia Machado de OLIVEIRA ${ }^{3}$ \\ Denecir de Almeida DUTRA ${ }^{4}$ \\ Eduardo Borba NEVES ${ }^{5}$
}

\section{RESUMO}

Recebido em: 26/05/2014 - Aprovado em: 30/06/2014 - Disponibilizado em: 30/07/2014

\begin{abstract}
Objetivou-se caracterizar a utilização do PICC em uma UTINEO no Estado do Paraná. Tratou-se de um estudo de campos descritivo retrospectivo. Compuseram a amostra 22 profissionais e 69 formulários preenchidos por enfermeiros da Instituição em um período de dois anos. Quanto à permeabilização do PICC, 90,90\% $(\mathrm{n}=20)$ utilizam solução fisiológica a $0,9 \%$. Ressalta-se que $68,18 \%(\mathrm{n}=15)$ relataram ter algum tipo de dúvida quanto à manipulação do PICC. Identificou-se que a maioria $(54,54 \%$; $n=12)$ nunca recebeu nenhum tipo de treinamento sobre o PICC. Percebeu-se um número significativo de RN de extremo baixo peso. No que se refere aos principais diagnósticos houve maior prevalência de Prematuridade. As veias utilizadas foram Cefálicas $(27,53 \%$; $n=19)$ e Basílica mediana $(24,63 \%$; $n=16)$. Dentre as principais soluções infundidas prevaleceu a antibioticoterapia com $(40,57 \%$; $n=28)$. Em 15,94\% ( $n=11)$ dos formulários foram constatadas anotações sobre o uso de manobras de desobstrução pelos enfermeiros com o uso de Vitamina C diluída em solução fisiológica $0,9 \%$. Quanto à retirada do PICC, evidenciou-se que $(13,04 \% ; n=9)$ se deu por rompimento, $(7,24 \% ; n=5)$ obstrução, $(5,79 \% ; n=4)$ infecção não especificada no formulário, $(4,34 \% ; n=3)$ infiltração e $(2,89 \%$; $\mathrm{n}=2)$ alta da UTINEO. Conhecer o perfil do RN submetido ao PICC é essencial para prever situações de risco e afirmar a indicação e tempo de uso de forma mais eficaz. O PICC foi inserido em sua maioria em RN's prematuros e de baixo peso. Portanto, cabe discutir a imedita indicação do PICC nesta condição clínica, com vista a favorecera terapêutica proposta a cada caso.
\end{abstract}

Palavras-chave: Enfermeiro; Terapia Intensiva Neonatal; Cateter Central de Inserção Periférica; Educação permanente; Tecnologia assistencial.

\section{ABSTRACT}

This study aimed to characterize the use of PICC in a UTINEO in Paraná State. This was a retrospective descriptive field. Sample thus included 22 professionals and 69 forms completed by nurses of the institution in a period of two years. As for the permeabilization of the PICC, $90.90 \%(n=20)$ using $0.9 \%$ saline. It is noteworthy that $68.18 \%(\mathrm{n}=15)$ reported having some kind of doubt about the handling of the PICC. It was found that the majority $(54.54 \%, \mathrm{n}=12)$ never received any training on the PICC. It was noticed a significant number of extremely low birth weight infants. With regard to the main diagnostic higher prevalence of prematurity. The cephalic veins were used $(27.53 \%, \mathrm{n}=19)$ and Church median $(24.63 \%, \mathrm{n}=16)$. Among the main solutions infused prevailed with antibiotics $(40.57 \%, \mathrm{n}=28)$. In $15.94 \%$ ( $n=11$ ) forms of the notes on the use of maneuvering clearance by nurses with the use of Vitamin $\mathrm{C}$ diluted in $0.9 \%$ saline were found . The withdrawal of the PICC, it is demonstrated that $(13.04 \%, \mathrm{n}=9)$ by breaking occurred $(7.24 \%, \mathrm{n}=5)$ obstruction $(5.79 \%, \mathrm{n}=4)$ infection unspecified in the form $(4.34 \%, \mathrm{n}=3)$ and infiltration $(2.89 \%, \mathrm{n}=2)$ of high UTINEO. Knowing the profile of newborns submitted to the PICC is essential to predict risk situations and affirm the statement and use time more effectively. The PICC was inserted mostly in RN's premature and low birth weight. Therefore, it discuss the imedita indication of PICC in this condition, overlooking the proposed therapeutic favored in each case.

Keywords: Nurse; Neonatal Intensive Care Unit; Peripherally Inserted Central Catheter; Continuing education; Assistive technology.

\footnotetext{
${ }^{1}$ Enfermeira. Centro Universitário Campos de Andrade. Curitiba -PR.

${ }^{2}$ Enfermeiro. Esp. Saúde Pública. Docente Centro Universitário Campos de Andrade. Curitiba -PR.

${ }^{3}$ Enfermeira. Mestre em Cirurgia. Docente Centro Universitário Campos de Andrade. Curitiba -PR.

${ }^{4}$ Geógrafo. Doutor em Geoggrafia da Saúde. Docente Centro Universitário Campos de Andrade. Curitiba -PR.

${ }^{5}$ Fisioterapeuta. Doutor em Saúde Pública. Docente Centro Universitário Campos de Andrade. Curitiba -PR.
} 


\section{INTRODUÇÃO}

O avanço tecnológico na Neonatologia vem beneficiando os recém-nascidos que carecem de um acesso venoso seguro. Este procedimento necessita de uma atenção específica, pois apresentam peculiaridades práticas. Desta forma é importante que o profissional de saúde responsável pela instalação desse acesso tenha os conhecimentos necessários em relação à fisiologia e anatomia da rede venosa(Rodrigues, Chaves e Cardoso, 2006).

Garantir o acesso venoso seguro é um desafio constante da equipe de en-fermagem, pois influencia na sobrevida e no prognóstico dos neonatos. Para a sobrevivência de recémnascidos de muito baixo peso é substancial um suporte nutricional adequado que supra as demandas de crescimento e desenvolvimento, carecendo dispor de um acesso vascular seguro e adequado. Isto promove aos profissionais que se ocupam da assistência neonatal a melhorar os métodos de prover acesso vascular seguro para esse grupo vulnerável(Costa et al., 2012).

\section{O Peripherally Inserted Central}

Catheter ou cateter central de inserção peirféica (PICC) traz benefícios ao cuidado do paciente, mas também pode conduzir a complicações. Este é um dispositivo inserido através de uma veia superficial da extremidade do corpo, que com o auxílio de uma agulha introdutora progride até a veia cava superior ou inferior, apresentando características de um cateter central. É considerado um procedimento de alta complexidade técnica que exige conhecimentos específicos(Johann et al., 2012).

Atualmente o PICC é o dispositivo mais utilizado nas Unidades de Terapia Intensiva Neonatal (UTINEO), sobretudo por suas vantagens, que são: inserção menos traumática; possibilidade de administração de medicamentos irritantes e/ou vesicantes; disponibilidade de vários acessos venosos para a seleção; diminuição do estresse causado pelas punções venosas; redução de custo; melhor evolução clínica, entre outras. As desvantagens estão relacionadas ao tipo de material e característica do cateter; restrição do movimento; fixação e a necessidade de cuidados diários(Camargo, 2007).

Observa-se que o PICC é utilizado em um amplo universo que abrange os cateteres centrais. No entanto, seu uso deve levar em consideração as especificidades inerentes ao dispositivo e ao perfil do paciente submetido ao procedimento de inserção(Noelly e Silva, 2013).

Desta forma, a carência de estudos sobre a utilizaçãodo PICC em UTINEO é um a variável que deve ser considerada, sobretudo, pelo PICC se tratar de um cateter de alto custo, com indicação especifica e que traz grandes benefícios aos pacientes, mas também malefícios em situações em que a 
equipe que o insere e manuseia não é adequadamente preparada. Pode ser considerado um procedimento simples, mas requer treinamento especificio conforme aponta a resolução ${ }^{\circ}$ 258/2001 do Conselho Federal de Enfermagem (COFEN)(Reis et al., 2011).

Portanto, a ausência de estudos aprofundados sobre o perfil dos pacientes que utilizam o PICC e demais variáveis como tempo de permamencia, patologia associada e formação deficitária dos profisisonais que manuseiam este dispositivo foi à problemática abordada neste estudo. Desta forma, o objetivo foi caracterizar a utilização do PICC em uma UTINEO no Estado do Paraná.

\section{MATERIAL E MÉTODOS}

A pesquisa foi realizada em um Hospital e Maternidade na cidade de São José dos Pinhais, estado do Paraná, que dispõe de uma unidade de UTI Neonatal com capacidade para 10 leitos.

Para o alcance dos objetivos a pesquisa foi dividida em duas fases para a coleta de dados: a primeira fase foi constituída do levantamento do perfil da equipe de Enfermagem mediante aplicação de questionário. Responderam ao questionário 22 dos 27 componentes da equipe de Enfermagem, considerando todos os turnos. Destaca-se que3 técnicos em enfermagem estavam em período de férias e outros 2 se recusaram a participar da pesquisa.

A segunda fase consistiu no levantamento de dados num período de dois anos (2011 a 2013), por meio de análise documental retrospectiva. Esta análise foi realizada em formulário existente na unidade, elaborado por Enfermeiros, denominado "Anotações e Registros de PICC', com a finalidade de documentar, acompanhar e avaliar todos os cateteres PICC inseridos na referida UTI, bem como o levantamento de dados sobre PICC a partir do banco de dados da Comissão de Infecção Hospitalar (CCIH) do referido Hospital.

Compuseram a amostra inicial 128 formulários preenchidos após a inserção do PICC. Foram critérios de inclusão dos formulários: preenchimento adequado e completo, acompanhamento do paciente em uso do cateter desde a inserção até a sua remoção. Desta forma, a amostra final foi composta por 69 formulários.

Consideraram-se as seguintes variáveis analíticas dos dados coletados: tempo de atuação da equipe na UTI neonatal, conhecimento sobre o PICC, capacitação dos Enfermeiros, peso do RN, principais diagnósticos, veias mais utilizadas para inserção, condições do acesso venoso, calibre do cateter utilizado, progressão, posicionamento, permanência em dias, curativos e motivo da remoção.

O estudo seguiu o que preconiza a Resolução número 196/96 do Conselho 
Nacional de Saúde que trata de pesquisas envolvendo Seres Humanos. O estudo foi aprovado pelo Parecer Consubstanciado número 339.631 de 23/07/2013 pelo Comitê de Ética em Pesquisa do Centro Universitário Campos de Andrade. Todos os sujeitos que concordaram em participar da pesquisa declararam sua vontade pelo Termo de Consentimento Livre e Esclarecido.

\section{RESULTADOS}

\section{Perfil da equipe de Enfermagem}

A equipe de enfermagem da UTI neonatal é constituída por $18,18 \% \quad(n=4)$ enfermeiras, em que 2 são habilitadas técnica e legalmente para a inserção do cateter PICC e por $81,81 \% \quad(n=18)$ de técnicos em enfermagem.

Quanto ao sexo dos profissionais de enfermagem entrevistados, observou-se a totalidade do sexo feminino. Quanto à idade observou-se o predomínio da faixa etária de mais de 30 anos $(59,09 \% ; n=13)$ seguido de 21 a 30 anos $(40,90 \% ; n=9)$. No que se refere à permanência no setor específico de UTI neonatal, observou-se o predomínio de menos de 1 ano $(36,36 \% ; n=8), 1$ a 3 anos $(31,81 \%$; $\mathrm{n}=7)$, de 3 a 5 anos $(18,18 \% ; \mathrm{n}=4)$ e mais de 5 $\operatorname{anos}(13,63 \% ; n=3)$.

\section{Conhecimentos específicos sobre PICC}

Evidenciou-se que $100 \%(n=22)$ das entrevistadasmarcaram que a seringa mais apropriada para realização de flush é a de 10 e $20 \mathrm{ml}$. Observou-se que da mesma forma demarcaram não utilizar hemoderivados devido ao alto risco de obstrução do cateter. Quanto à permeabilização do PICC, 90,90\% $(\mathrm{n}=20)$ utilizam solução fisiológica a $0,9 \%$. Os demais 9,09\% $(\mathrm{n}=2)$ responderam que a solução mais adequada seria a solução glicosada a $5 \%$.

Em relação ao tempo de permanência apenas $13,63 \% \quad(n=3)$ responderam que a utilização do PICC é de um mês. A maioria $(86,36 \%$; n=19) marcou a utilização por tempo indeterminado.

Ressalta-se que $68,18 \%(\mathrm{n}=15)$ das entrevistadas relataram ter algum tipo de dúvida quanto à manipulação do cateter. Quando questionadas quanto à conduta frente estas dúvidas 59,09\% (n=13) se reportam a outro profissional capacitado ou buscam informações em outros meios $(45,45 \%$; $n=$ 10).

Identificou-se que a maioria $(54,54 \%$; $\mathrm{n}=12$ ) nunca recebeu nenhum tipo de treinamento sobre o PICC. Outros $(45,45 \%$; $\mathrm{n}=10$ ) relataram ter participado de treinamentos em outras instituições e algumas vezes orientações da enfermeira do setor, além das enfermeiras que já possuem capacitação. 
Caracterização dos RN submetidos à PICC na UTI neonatal

Percebeu-se um número significativo de RN de extremo baixo peso. No que se refere aos principais diagnósticos, percebeuse a maior prevalência de Prematuridade, seguido de Asfixia e Distress Respiratório. A Tabela 1 e 2 apresenta estas variáveis.

Tabela 1. Peso dos RNs submetidos ao PICC, São José dos Pinhais, 2013.

\begin{tabular}{ccc}
\hline Peso & n & \% \\
\hline 500 a $1000 \mathrm{~g}$ & 08 & 11,59 \\
1001 a $1500 \mathrm{~g}$ & 13 & 18,84 \\
1501 a $2000 \mathrm{~g}$ & 21 & 30,43 \\
2001 a $2500 \mathrm{~g}$ & 10 & 14,49 \\
2501 a $3000 \mathrm{~g}$ & 08 & 11,59 \\
Acima de 3000 g & 09 & 13,04 \\
TOTAL & $\mathbf{6 9}$ & $\mathbf{1 0 0}$ \\
\hline
\end{tabular}

Tabela 2. Diagnóstico dos RN submetidos à PICC, São José dos Pinhais, 2013.

\begin{tabular}{clc}
\hline Diagnóstico & $\mathbf{n}$ & \% \\
\hline Prematuridade & 31 & 44,92 \\
Infecção Neonatal & 04 & 5,79 \\
Asfixia & 21 & 30,43 \\
Distress Respiratório & 09 & 13,04 \\
Síndrome da Aspiração & 05 & 7,24 \\
Meconial & & \\
Outros & 08 & 11,59 \\
TOTAL & $\mathbf{6 9}$ & $\mathbf{1 0 0}$ \\
\hline
\end{tabular}

\section{Inserção e manutenção do PICC}

Referente aos dados da inserção do cateter percebeu-se que as veias utilizadas foram: Cefálica $(27,53 \% ; n=19)$, Basílica mediana $(24,63 \% ; n=16)$, Safena $(15,94 \%$; $\mathrm{n}=10)$, Basílica $(13,04 \% ; \mathrm{n}=9)$, Jugular externa $(10,14 \% ; n=7)$, Axilar $(8,69 \% ; n=6)$ e outras $(2,89 \% ; n=2)$.

Com relação ao tempo aproximado de procedimento, a maioria $(47,82 \% ; n=33)$ foi de até uma hora e $(18,84 \% ; n=13)$ mais de uma hora. A condição de acesso se mostrou difícil em (33,33\%; $\mathrm{n}=23)$ e fácil em $(66,66 \% ; n=46)$. Assim, o número de tentativas de punções variou de 1 a 4 , sendo que 1 tentativa foi a mais prevalente $(46,37 \%$; $\mathrm{n}=32)$. O antisséptico utilizado em $(95,65 \%$; $\mathrm{n}=66$ ) foi Álcool $70 \%$.

Observou-se uso de sedação em apenas (13,04\%; n=9) dos RN com uso de Midazolan. Percebeu-se que em relação à data de inserção a maioria $(69,56 \% ; n=48)$ tinha menos de 7 dias de vida. A Tabela 3 apresenta de forma mais detalhada estas variáveis.

Tabela 3. Dados referentes à inserção do PICC, São José dos Pinhais, 2013

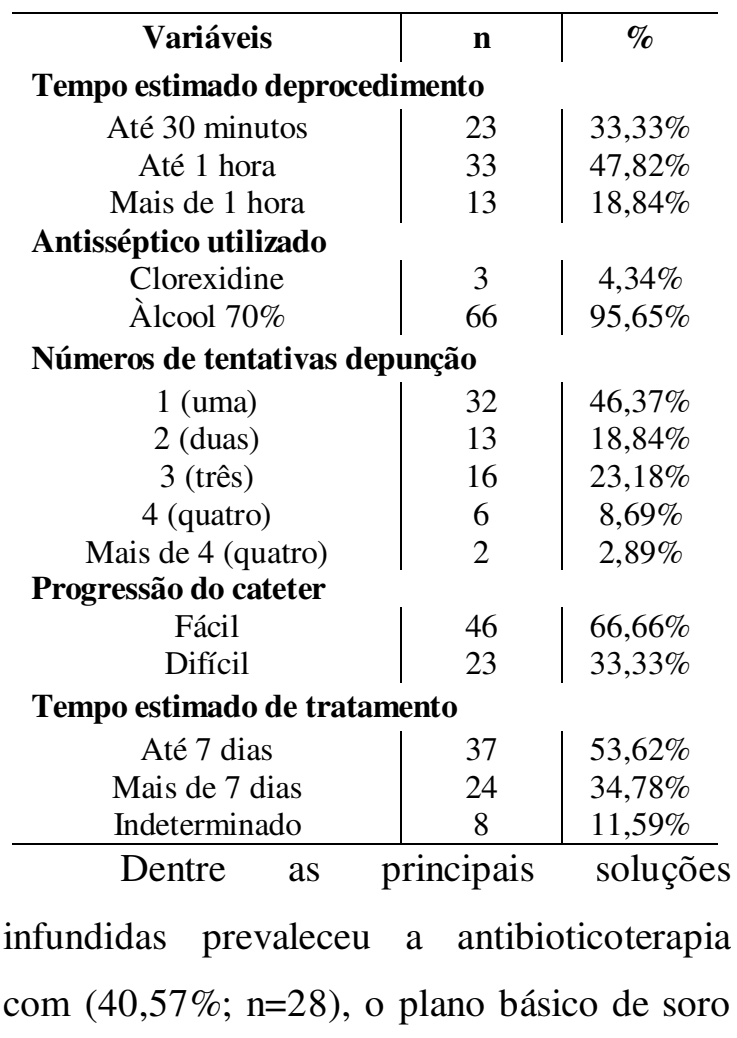


(24,63\%; n=17), Nutrição Parenteral $(23,18 \% ; n=16)$ e drogas vasoativas $(11,59 \%$; $\mathrm{n}=8$ ). Em relação ao motivo da troca do curativo a maioria $(68,11 \% ; n=47)$ se deu devido à presença de sangue e exudatos.

Em 15,94\% (n=11) dos formulários foram constatadas anotações sobre o uso de manobras de desobstrução pelos enfermeiros e todos relataram o uso de Vitamina c diluída em solução fisiológica $0,9 \%$ para o procedimento.

Quanto à retirada do PICC, percebeuse que a maioria se deu devido ao término do tratamento. No entanto, evidenciou-se que $(13,04 \% ; n=9)$ foi por rompimento, $(7,24 \%$; n=5) obstrução, $(5,79 \%$; n=4) infecção não especificada no formulário, $(4,34 \% ; \mathrm{n}=3)$ infiltração e $(2,89 \% ; n=2)$ alta da UTINEO. Em $(5,79 \% ; n=4)$ dos formulários constavam a observação de retirada de cateter acidental, 1 pela equipe de enfermagem ao manuseio, 1 pelo técnico de RX durante posicionamento para o exame e 2 pelo manuseio da mãe ao amamentar. Em 2 destes 4 casos o cateter tinha sido inserido com menos de 12 horas.

\section{DISCUSSÃO}

Quanto à caracterização relativa ao sexo, prevaleceu o feminino na totalidade dos sujeitos entrevistados. Tal fato está em concordância com a historicidade da Enfermagem, com o prodominio de mulheres na assistência ao paciente. Sugere que na
UTINEO a ausência de profissionais do sexo masculino se dê principalmente ao arquétipo atribuído as mulheres, sobretudo, em se tratando de recém nascidos(Oguisso, 1990; Gardenal et al., 2002; Almeida et al., 2004; Martins et al., 2006).

A respeito do tempo de formação, prevaleceu tempo inferior a 1 ano, seguido de 1 a 3 anos. Ressalta-se que o tempo de formado pode ser considerado um indicativo de experiência e maturidade(Vendrúsculo, 1990; Martins et al., 2006). O tempo de formação pode favorecer que o enfermeiro desenvolva suas habilidades e competenciasassistenciais, sobretudo, em procedimentos de maior complexidade, como a inserção do PICC.

Relativo aos conhecimentos especificos sobre PICC evidenciou-se que a totalidade dos sujeitos assinalou dúvida entre o volume da seringa apropriada para a realização do flush. Neste sentido, (Margotto, 2006) destaca que quanto menor o volume da seringa, maior pressão poderá ser gerada, expondo desta forma o cateter ao risco de ruptura. Portanto, sugere-se não utilizar seringas com volume de 1,3 ou 5 mililitros. No presente estudo os sujeitos referiram utilizar seringas de 10 e 20 mililitros, técnica considerada correta e sem riscos ao paciente.

Quanto à permeabilização do PICC a solução fisiológica $0,9 \%$ foi citada pela maioria. Os demais $(9,09 \%, \quad n=2)$ responderam que a solução mais adequada 
seria a solução glicosada a 5\%. É recomendável lavar o cateter com solução fisiológica $0,9 \%$ após a infusão de medicamentos. Em casos de infusão contínua a lavagem deve ser realizada a cada seis (6) horas(Freitas e Nunes, 2009).Em relação ao tempo de permanência $13,63 \%$ responderam ser de um mês. A maioria $(86,36 \%)$ marcou a utilização por tempo indeterminado. Não há tempo determinado para a permanência do PICC, no entanto, o Centers for Disease Control (CDC) recomenda a utilização por oito (8) semanas(Câmara, Tavares e Chaves, 2012).

O tempo de permanência do PICC foi em média 7 dias (3-28 dias). Em um estudo retrospectivo durante 24 meses o período médio de uso foi de 45 dias (14-300 dias)(Delarbre et al., 2014). Já no estudo de Camargo(Camargo, 2007) o tempo médio foi 8,9 dias. O tempo de permanência do PICC é uma variável que pode ser associada ao maior risco por seu maior tempo de manipulação pela equipe.

Ressalta-se que 68,18\% (n=15) das entrevistadas relataram ter algum tipo de dúvida quanto à manipulação do cateter e a maioria $(54,54 \%)$ nunca recebeu nenhum tipo de treinamento sobre o PICC. Neste sentido, ser apto a reconhecer riscos e complicações é dependente de cada profissional, e buscar capacitação e informações atualizadas é algo inevitável para a agarantia da segurança do paciente. Ainda, há necessidade de treinamentos para toda a equipe de enfermagem deve ser considerada, visto que a prática de terapia intravenosa com o uso do PICC é crescente no Brasil(Secoli e Jesus, 2008).

Dentre os RN's submetidos ao PICC, prevaleceram os de baixo peso diagnosticados com prematuridade e asfixia neonatal. A prematuridade resulta em imaturidade anatômica e fisiológica, próprias deste grupo populacional, resultando em maior susceptibilidade a distúrbios respiratórios(Michelin et al., 2013). Estima-se que nos Estados Unidos, nascem 60.000 prematuros com peso inferior a $1.500 \mathrm{~g}$. Dados do Brasil emitidos pelo Sistema nacional de Registro de Nascidos Vivos (SINASC) apontam um aumento nos nascimentos prematuros nas últimas décadas(Lopes e Mendes, 2014).

São considerados nascidos prematuros aqueles que não completaram 37 semanas de gestação e baixo peso ao nascer (inferior a 2500g). Destaca-se que este grupo apresenta maior risco de mortalidade, cerca de um terço dos óbitos neonatais são decorrentes da prematuridade. No Brasil a taxa de mortalidade neste grupo está entre 7,9 e 14,2\%(Lopes e Mendes, 2014).

A asfixia neonatal é um distúrbio na troca gasosa, resultando em hipoxemia e hipercapnia, levando a predisposição á sepse devido ao quadro de neutropenia e redução de reservas medulares de neutrófilos(Souza 
Santos et al., 2014). Portanto, evidenciou-se que estes RN's diagnosticados com prematuridade, baixo peso e asfixia neonatal foram os mais submetidos ao PICC.

Quanto aos vasos para a inserção do cateter, os dados corroboram com a literatura, sendo as principais escolhas as veias basílica e cefálica. Estas apresentam menor número de válvulas, maior calibre, anatomia favorável e facilitam a realização e troca de curativos (Baggio, M. A., Bazzi, F. e Bilibio, C. A. C., 2010; Delarbre et al., 2014).

Em relação ao tempo aproximado de procedimento, a maioria foi de até uma hora. O número de tentativas de punção foi de uma vez em 46,37\% dos casos. A condição de acesso se mostrou fácil em $68 \%$ dos casos. Entre as soluções infundidas prevalereram a antibióticos e nutrição parenteral. Resultado semenlhante ao descrito por (Delarbre et al., 2014) em seu estudo, com $(n=47,52 \%)$ de antibioticoterapia, $(\mathrm{n}=34,38 \%)$ quimioterapia e $(n=5,5 \%)$ para nutrição parenteral.

Relativo ao número de tentativas de punção para a inserção da PICC, 69,55\% dos enfermeiros obteve sucesso com 1 ou 3 tentativas. Outros estudos apresentaram uma variação média de 2,7 a 3,4 tentativas, com sucesso na primeira tentativa entre 21,6 a $47,0 \%$, inferiores a retratada no presente estudo(Montes et al., 2011). Em um estudo realizado por (Montes et al., 2011) o número de tentativas foi em média de 3,1+2,5 vezes.

$\mathrm{Na}$ variável tempo estimado de tratamento com o uso do PICC, a maioria $(53,62 \%)$ dos RN's utilizou por 7 dias. Em $34,78 \%$ dos casos o uso de prolongou por mais de 7 dias. Em estudo semelhante observou-se o tempo de permanência médio de 8,8 $\pm 6,1$ dias (mínimo de 2 horas e máximo de 33 dias). A maioria dos cateteres permaneceu inserida entre seis a dez dias (Montes et al., 2011).

Observou-se uso de sedação em apenas $13 \%$ dos RN com uso de Midazolan. A inserção do PICC é um procedimento extremamente doloroso e estressante ao RN, e necessita que este esteja calmo e tranquilo para o sucesso na progressão do cateter. Portanto, é relevante solicitar a equipe médica a analgesia ou sedação antes da inserção do cateter(Costa et al., 2012).Em outro estudo citou-se o uso de anestesia geral $(4,4 \%)$ e uso de anestésicos tópicos $(95,6 \%)($ Delarbre et al., 2014).

Em 15,94\% (n=11) dos formulários foram constatadas anotações sobre o uso de manobras de desobstrução pelos enfermeiros e todos relataram o uso de Vitamina c diluída em solução fisiológica $0,9 \%$ para o procedimento. Ressalta-se que a obstrução do PICC pode ocorrer por formação de trombos na luz do do cateter, formação de placas de fibrina e por precipitação de dorgas infundidas. Nestes casos, a primeira ação é buscar a acausa da obstrução. Após, sugere-se manobras de reposicionamento, administração de ativadores de plasminogênio tissular ou 
outras soluções com o uso de pressão negativa ou flush(Costa et al., 2012).

Em situações que não é possível a desobstrução do cateter se faz necessário a retirada do mesmo. No presente estudo a maioria das retiradas se deu devido ao término do tratamento proposto aos $\mathrm{RN}$. No entanto, 13,04 foram motivados por rompimento, seguido de 7,24\% obstrução e $5,79 \%$ infecção. Percebe-se que a o rompimento do cateter, obstrução e infecção são as complicações mais prevalentes(O'grady et al., 2011; Delarbre et al., 2014).

As possíveis causas do rompimento incluem o excesso de pressão no flush gerado por seringas com calibre menor de 10 mililitros. Outros fatores que podem influenciar são as mudanças na composição da equipe de enfermagem e profissionais em fase de treinamento(Margotto, 2006).

Em 5,79\% dos formulários constavam a observação de retirada acidental do cateter. Entre estes, 1 pela equipe de enfermagem ao manuseio, 1 pelo técnico de RX durante posicionamento para o exame e 2 pelo manuseio da mãe ao amamentar. Em 2 destes 4 casos o cateter tinha sido inserido com menos de 12 horas. A retirada acidental pode ser causada por fixação inadequada ou insuficiente, sangramento excessivo e excesso no compirmento da extensão externa do cateter(Paulson e Miller, 2008; Baggio, M. A., Bazzi, F. C. D. S. e Bilibio, C. A. C., 2010; Costa et al., 2012).
A manutenção diária do cateter deve ser feita por pessoal capacitado e treinado, sobretudo, curativos realizados somente por enfermeiro devidamente habilitado. A troca do curativo possui as funções de proteger o local da inserção e evitar deslocamento(Baiocco e Silva, 2010).

\section{CONCLUSÕES}

Observou-se que grande parte da equipe de Enfermagem apresenta lacunas no conhecimento teórico e prático sobre o PICC. Neste sentido, a educação permanente, adoção de protocolos mais abrangentes e a correta utilização instrumentos que visam mensurar a eficácia de procedimentos devem ser considerados.

Destaca-se que a correta manutenção e manuseio do cateter evita infecções e perdas dos mesmos, uma vez que, estes apresentam um alto custo para as instituições hospitalares, e consequente risco ao RN.

Neste sentido, conhecer o perfil do RN submetido ao PICC é essencial para prever situações de risco e afirmar a indicação e tempo de uso de forma mais eficaz. O PICC foi inserido em sua maioria em RN's prematuros e de baixo peso. Portanto, cabe discutir a imedita indicação do PICC nesta condição clínica, com vista a favorecera terapêutica proposta a cada caso. 


\section{REFERÊNCIAS}

ALMEIDA, M. C. P. et al. Perfil da demanda dos alunos da pós-graduação scricto sensu da escola de enfermagem de Ribeirão Preto, da Universidade de São Paulo. Rev Latino-am Enfermagem, v. 12, n. 2, p. 153-61, 2004.

BAGGiO, M. A.; BAZZI, F.; BILIBIO, C. A. C. Cateter central de inserção periférica: descrição da utilização em UTI Neonatal e Pediátrica. Rev Gaúcha Enferm, v. 31, n. 1, p. 70-6, 2010.

BAGGIO, M. A.; BAZZI, F. C. D. S.; BILIBIO, C. A. C. Cateter central de inserção periférica: descrição da utilização em UTI Neonatal e Pediátrica. Revista Gaúcha de Enfermagem, v. 31, n. 1, 2010.

BAIOCCO, G. G.; SILVA, J. A utilização do cateter central de inserção periférica (CCIP) no ambiente hospitalar. Rev. Latino-Am. Enfermagem, v. 18, n. 6, p. 1131-1137, 2010.

CÂMARA, S. M. C.; TAVARES, T. J. L.; CHAVES, E. M. C. Cateter venoso de inserção periférica: análise do uso em recém-nascidos de uma unidade neonatal pública em Fortaleza. Revista da Rede de Enfermagem do Nordeste-Rev Rene, v. 8, n. 1, p. 327, 2012. ISSN 2175-6783.

CAMARGO, P. Procedimento de inserção, manutenção e remoção do cateter central de inserção periférica em neonatos [dissertação]. São Paulo: Escola de Enfermagem, Universidade de São Paulo, 2007.

COSTA, S. P. et al. Prevalência e motivos de remoção não eletiva do cateter central de inserção periférica em neonatos. Revista Gaúcha de Enfermagem, v. 33, n. 3, p. 126-133, 2012. ISSN 1983-1447.

DELARBRE, B. et al. Introduction of the use of a pediatric PICC line in a French University Hospital: Review of the first 91 procedures. Diagnostic and interventional imaging, v. 95, n. 3, p. 277-281, 2014. ISSN 2211-5684.

FREITAS, E. M.; NUNES, Z. B. O enfermeiro na práxis de cateter central de inserção periférica em neonato. Revista Mineira de Enfermagem, v. 13, n. 2, p. 215-224, 2009. ISSN 1415-2762.

GARDENAL, C. L. C. et al. Profile of nurses working in institutions where care to pregnant, parturient and puerperal women was given in the city of Sorocaba-SP, Brazil (1999). Revista Latino-Americana de Enfermagem, v. 10, n. 4, p. 478-484, 2002. ISSN 0104-1169.
JOHANN, D. A. et al. Peripherally inserted central catheter care in neonates: an integrative literature review. Revista da Escola de Enfermagem da USP, v. 46, n. 6, p. 1503-1511, 2012. ISSN 0080-6234.

LOPES, S. A.; MENDES, C. M. C. Prematuridade e assistência pré-natal em Salvador. Revista de Ciências Médicas e Biológicas, v. 12, n. 4, p. 460-464, 2014. ISSN 2236-5222.

MARGOTTO, P. R. Assistência ao recém-nascido de risco. Hospital Anchieta, Brasília, 2006.

MARTINS, C. et al. Perfil do enfermeiro e necessidades de desenvolvimento de competência profissional. Texto Contexto Enferm, v. 15, n. 3, p. 14, 2006.

MICHELIN, A. S. et al. Hemodynamic effects of noninvasive ventilation with facial mask in premature infants. Fisioterapia e Pesquisa, v. 20, n. 4, p. $367-$ 372, 2013. ISSN 1809-2950.

MONTES, S. et al. Ocorrência de complicações relacionadas ao uso de cateter venoso central de inserção periférica (PICC) em recém-nascidos. Enfermería global, v. 24, n. 4, p. 10-18, 2011.

NOELLY, C.; SILVA, C. M. P. Fatores associados à infecção pelo uso do cateter central de inserção periférica em Unidade de Terapia Intensiva Neonatal. Rev Esc Enferm USP, v. 47, n. 3, p. 547-54, 2013.

O'GRADY, N. P. et al. Guidelines for the prevention of intravascular catheter-related infections. Clinical infectious diseases, v. 52, n. 9, p. e162-e193, 2011. ISSN 1058-4838.

OGUISSO, T. Perfil do enfermeiro de unidades ambulatoriais do INAMPS no Brasil. Rev. esc. enferm. USP, v. 24, n. 1, p. 77-92, 1990. ISSN 00806234.

PAUlSON, P. R.; MILLER, K. M. Neonatal peripherally inserted central catheters: recommendations for prevention of insertion and postinsertion complications. Neonatal Network: The Journal of Neonatal Nursing, v. 27, n. 4, p. 245-257, 2008. ISSN 0730-0832.

REIS, T. et al. O uso do cateter epicutâneo na clientela neonatal de um hospital público estadual: estudo retrospectivo. Rev Enferm UERJ, v. 19, n. 4, p. 5927, 2011.

RODRIGUES, Z. S.; CHAVES, E. M. C.; CARDOSO, M. Atuação do enfermeiro no cuidado com o cateter 
central de inserção periférica no recém-nascido. Revista Brasileira de Enfermagem, v. 59, n. 5, p. 626-629, 2006. ISSN 0034-7167.

SECOLI, S. R.; JESUS, V. C. Complicações acerca do cateter venoso central de inserção periférica (PICC). Ciência, cuidado e saúde, v. 6, n. 2, p. 252-260, 2008. ISSN 1984-7513.

SOUZA SANTOS, A. P. et al. Diagnósticos de enfermagem de recém-nascidos com sepse em uma Unidade de Terapia Intensiva Neonatal. Rev. LatinoAm. Enfermagem, v. 22, n. 2, p. 255-61, 2014.

VENDRÚSCULO, D. A criança inserida no currículo de graduação: o ensino da assistência de enfermagem. Ribeirão Preto (SP): USP/EERP, 1990. 